\title{
Magnitude and sign scaling in power-law correlated geoelectrical time series measured in southern Italy
}

\author{
L. Telesca and V. Lapenna \\ Institute of Methodologies for Environmental Analysis, National Research Council, C.da S. Loja, 85050 Tito (PZ), Italy \\ Received: 30 June 2004 - Revised: 25 October 2004 - Accepted: 26 October 2004 - Published: 1 November 2004 \\ Part of Special Issue "Precursory phenomena, seismic hazard evaluation and seismo-tectonic electromagnetic effects"
}

\begin{abstract}
Recently, it has been proposed a new method to investigate the linear/nonlinear properties of a time series by decomposing it into two sub-series: magnitude and sign sub-series (Ashkenazy et al., 2003). The long-range correlation properties of the magnitude and sign sub-series relate to nonlinear and linear behavior of the original time series respectively. We apply this method to the hourly time variability of geoelectrical signals, measured from January 2001 to September 2002 by four stations installed in Basilicata region (southern Italy). Three stations (Giuliano, Marsico and Tito) are located in a seismic area, and one (Laterza) in a lowseismicity area. A site-dependence of the linear/nonlinear properties has been shown, revealing that different dynamics govern signals measured in seismic and low-seismicity areas.
\end{abstract}

\section{Introduction}

A wide class of geophysical systems presents complex dynamics associated with the existence of many components interacting over many time or space scales. These oftencompeting interactions can produce an output signal, which appears noisy and irregular, but in fact is characterized by power-law long-range correlations.

It has been shown that the time fluctuations of such system can be characterized by two components: magnitude, corresponding to the absolute value, and sign, corresponding to the direction (Ashkenazy et al., 2001). These two components reveal the underlying interactions of a system, and the resulting force of these interactions at each moment determines the magnitude and the direction of the fluctuations (Ashkenazy et al., 2003).

The temporal fluctuations of geoelectrical signals may be useful to monitor and understand many seemingly complex phenomena linked to seismic activity (Park, 1997; Johnston, 1997). They are the result of the interaction among very het-

Correspondence to: L. Telesca

(telesca@imaa.cnr.it) erogeneous and not well-known mechanisms, which can be influenced by the particular structure of the monitored zone (Patella et al., 1997). This means that local features can be mixed to the general ones so increasing the difficulty of rightly characterizing and interpreting the signal time variations.

Here we study the scaling properties of the magnitude and sign series of geoelectrical signals, measured in southern Italy, one of the most seismically active areas of the Mediterranean, from January 2001 to September 2002. This approach is able to discriminate the nonlinerity intrinsic in the signals, and seems to be promising in identifying sitedependent peculiarities in the time dynamics of geoelectrical time series.

\section{Geological and seismological settings}

The southern Apennine chain is an Adriatic-verging chain, built up from early Miocene to Pleistocene. It is mainly composed of sedimentary cover of platform and deep water environments, scraped off from the former Mesozoic Ligurian ocean, from the western passive margin of the Adriatic plate and from the Neogene-Pleistocene foredeep deposits of the active margin (Monaco and Tortorici, 1995; Giano et al., 1999). Thrusting in the frontal eastern part of the accretionary wedge is followed by back-arc extension in the rear to the west. One of the evidence of active extension along the Apennine axis is widely documented in the field (e.g. Val d'Agri, Vallo di Diano, etc.) and by seismicity (e.g. the 1980 Irpinia earthquake).

Quaternary folding and brittle deformations of subaerial slope deposits in the northern part of the Agri Valley have been studied through geophysical and structural analyses. The results revealed that the area underwent both transpressional and transtensional tectonics during Pleistocene times. On this basis, the valley appears to be a more complex structure than a simple extensional graben, as traditionally assumed in the literature. 

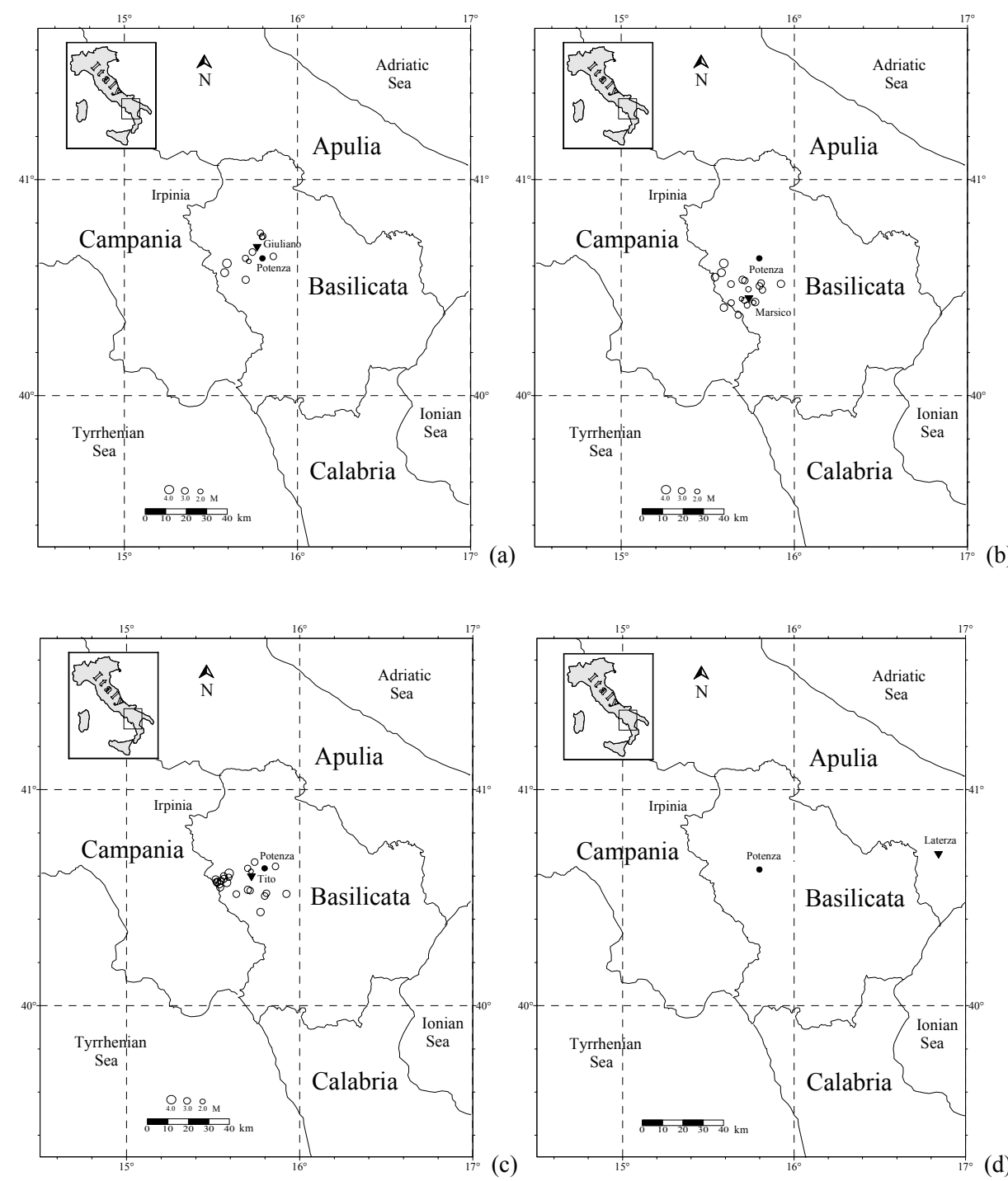

Fig. 1. Ubication of the geoelectrical monitoring stations and epicenters of the earthquakes satisfying the Dobrovol'skiy's rule in relation with the location of the measuring stations: (a) Giuliano, (b) Marsico, (c) Tito and (d) Laterza. The seismic data are extracted from the INGV (National Institute of Geophysics and Volcanology) seismic catalogue (http://www.ingv.it).

The architecture and the large-scale kinematics of the southern Apennines constructed a depth-extrapolated crosssection through the whole chain, from the Tyrrhenian Sea to the Adriatic (Apulian) foreland. Several tectonic units have been distinguished in the cross-section, moving from West to East, and from the top to the bottom of the chain. The Apulian carbonate platform has been incorporated by under plating at the base of the accretionary wedge. Restoration of the cross-section gave about $50 \%$ of shortening (corresponding to about $100 \mathrm{~km}$ ); not including the displacement related to the basal thrust of the Apennine units on the Apulian platform. Shortening could be at least twice considering that the deformed "Apulian" units should be present at the base of the whole accretionary wedge. Due to the time span estimated for the Apennine orogeny (about $20 \mathrm{Ma}$ ), a very high shortening rate may be calculated $(1 \mathrm{~cm} / \mathrm{y})$.
From the seismological point of view, the CampanoLucano sector of the Southern Apennines chain is one of the most active areas of the Mediterranean region. In particular, this area is characterised by a probability $>60 \%$ to generate at least one earthquake with $\mathrm{M}>3.5$ in a time span of 3 years (Martinelli and Albarello, 1997). In this region on February 1826 an earthquake, reaching up to VIII degree on the MCS scale (Alessio et al., 1995), hit the village of Tito where is located our prototype station. On 23 November 1980 (Ms=6.9), a large normal-faulting earthquake occurred in the nearby Irpinia area. Seismic activity occurred after the 1980 event consisted of medium intensity events $(M<5.5)$ located close to the border between Campania and Basilicata regions (Alessio et al., 1995).

The 5 May 1990 (MD=5.0, ING-National Institute of Geophysics) and the 26 May 1991 ( $\mathrm{MD}=4.7$ ) earthquakes took 

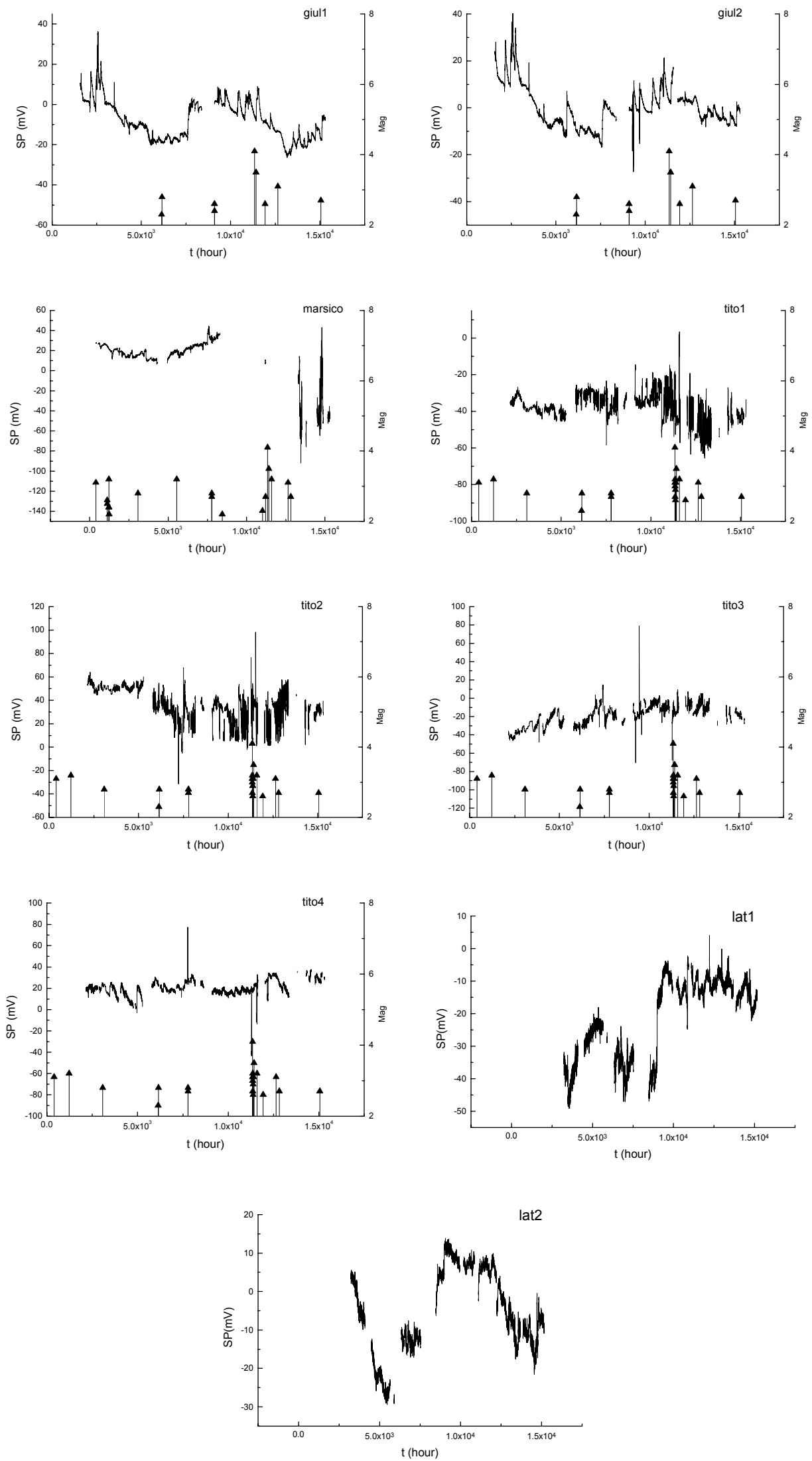

Fig. 2. Hourly variability of the 9 geoelectrical signals recorded at stations Giuliano, Marsico, Tito and Laterza, along with the occurrence of the earthquakes selected by means of the Dobrovol'skiy's rule. 
place in the north of Potenza town (Tertulliani et al., 1992). These events have been generated by a strike-slip fault system with WE direction, perpendicularly oriented toward the Apennine chain (Ekström, 1994), located in such a way to limit toward north and south two great seismogenetic faults that caused the 1857 Val d'Agri and 1980 Irpinia earthquakes respectively. These geological and seismological features, combined with a very low level of cultural noise, allow us to consider the selected area as an ideal outdoor laboratory to study the possible correlations between tectonic activity and anomalous patterns in SP signals. Since the area is also characterized by seismic quiescence periods, in principle, it is possible to test alarms and false alarms.

\section{Data}

Our data consist in nine geoelectrical time series recorded at four monitoring stations: Tito (Tito1, Tito2, Tito3 and Tito4), Giuliano (Giul1 and Giul2), Marsico and Laterza (Lat1 and Lat2). As far as the technical features of the experimental equipment are concerned, we refer the reader to Cuomo et al. (1997) and for the results of mono-and multi-parametric preliminary statistical analysis of the monitored variables to Di Bello et al. (1994). The Tito SP acquisition system consists of an array with 4 electrodes put at the corners of a $100 \mathrm{~m}$ side square. Station Giuliano is located just on a strike fault. It measures two SP signals. The dipoles are located parallel and perpendicular to the strike-fault and the distance between the probes is $100 \mathrm{~m}$ for the dipole oriented along the strike-fault direction (EW), and $80 \mathrm{~m}$ for the other dipole (NS) perpendicular to the fault. The dipole at station Marsico is $100 \mathrm{~m}$ long and oriented along NS direction. The Laterza dipole configuration is NW-SE $100 \mathrm{~m}$ and NE-SW $80 \mathrm{~m}$. The stations Tito, Giuliano and Marsico are located in seismic sites, while Laterza in a low-seismicity one.

The stress field produces cracks on the rock volumes triggering fluid pressure variations. As a result of this process we have an underground charge motion and, subsequently, we observe anomalies in the electrical field on the surface only if the preparation region is near the measuring station. It is necessary to discriminate the useful events (i.e. earthquakes responsible for significant geophysical variations in a rock volume of the investigated area) from all the seismicity that occurred in the area surrounding the measuring station. Therefore, from the whole seismicity, we selected only earthquakes that could be responsible for strain effects in the areas around the monitoring stations. We used the empirical formula introduced by Dobrovol'skiy (Dobrovol'skiy et al., 1979; Dobrovol'skiy, 1993): $r=10^{0.43 M}$, where $M$ is the magnitude and $r(\mathrm{~km})$ the radius of the area in which the effects of the earthquake are detectable. We considered only the earthquakes with $r$ greater than the distance between the epicenter and the measuring station. Figure 1 shows for each station the earthquakes that according to the Dobrovol'skiy's rule can affect the time variations of the measured signals. As we can clearly observe, the area monitored by station Laterza is characterized by a substantial absence of earthquakes.

Figure 2 shows the time variations of the self-potential signals along with the earthquakes occurred during the observation period. A striking feature is visible concerning especially the graphs of station Tito: an increased seismic activity, also with events with a relatively high magnitude $(M \geq 4.0)$, characterizes the SP time fluctuations in the temporal range $10^{4}<t<1.5 \cdot 10^{4}$. In this temporal range, the Tito SP fluctuations present the largest variability and irregularity. Giuliano signals are characterized by similar behaviour, with an increased number of spikes in the same temporal range as Tito. Marsico signal presents a long gap during the same time range, but its dynamics vary significantly between $1.3 \cdot 10^{4} \mathrm{~h}$ and $1.5 \cdot 10^{4} \mathrm{~h}$.

\section{Magnitude and sign decomposition}

Any power-law long-range correlated time series can be decomposed into two sub-series (Ashkenazy et al., 2001): given the series $x(i)$, define the increments as $\Delta x(i)=x(i+$ $1)-x(i)$; then the magnitude sub-series is the absolute value of the increments $m(i)=|\Delta x(i)|$, while the sign sub-series is the sign of the increments $s(i)=\operatorname{sgn}(\Delta x(i))$, therefore $\Delta x(i)=\operatorname{sgn}(\Delta x(i))|\Delta x(i)|$. Then, to identify the presence of correlations and their type in the sign and magnitude sub-series, the detrended fluctuation analysis (DFA) is performed. This method was originally proposed by Peng et al. (1995). It avoids spurious detection of correlations that are artefacts of nonstationarity, which often affect experimental data. Such trends have to be well distinguished from the intrinsic fluctuations of the system in order to find the correct scaling behaviour of the fluctuations. Very often we do not know the reasons for underlying trends in collected data and we do not know the scales of underlying trends. DFA is a method for determining the scaling behaviour of data in presence of possible trends without knowing their origin and shape (Kantelhardt et al., 2001).

The methodology operates on the time series $x(i)$, where $i=1,2, \ldots, N$ and $N$ is the length of the series. With $x_{\text {ave }}$ we indicate the mean value

$x_{\mathrm{ave}}=\frac{1}{N} \sum_{k=1}^{N} x(k)$.

The signal is first integrated

$y(k)=\sum_{i=1}^{k}\left[x(i)-x_{\mathrm{ave}}\right]$.

Next, the integrated time series is divided into boxes of equal length, $\mathrm{n}$. In each box a least-squares line is fit to the data, representing the trend in that box. The $y$ coordinate of the straight-line segments is denoted by $y_{n}(k)$. Next we detrend the integrated time series $y(k)$ by subtracting the local trend 
$y_{n}(k)$ in each box. The root-mean-square fluctuation of this integrated and detrended time series is calculated by

$F(n)=\sqrt{\frac{1}{N} \sum_{k=1}^{N}\left[y(k)-y_{n}(k)\right]^{2}}$.

Repeating this calculation over all box sizes, we obtain a relationship between $F(n)$, which represents the average fluctuation as a function of box size, and the box size $n$. If $F(n)$ behaves as a power-law function of $n$, the data present scaling:

$F(n) \propto n^{\alpha}$.

Under these conditions the fluctuations can be described by the scaling exponent $\alpha$, representing the slope of the linefitting $\log [F(n)]$ to $\log n$. For a white noise process, $\alpha=0.5$. If there are only short-range correlations, the initial slope may be different from 0.5 but will approach 0.5 for large window sizes. $0.5<\alpha$ indicates the presence of persistent longrange correlations, meaning that a large (compared to the average) value is more likely to be followed by large value and vice versa. $\alpha<0.5$ indicates the presence of antipersistent long-range correlations, meaning that a large (compared to the average) value is more likely to be followed by small value and vice versa. $\alpha=1$ indicates flicker-noise dynamics, typical of systems in a self-organized critical state. $\alpha=1.5$ characterizes processes with Brownian-like dynamics.

The correlation analysis of the magnitude and sign subseries consists of the following steps:

1. From the time series $\mathrm{x}(\mathrm{i})$ we derive the increments $\Delta x(i)$.

2. The magnitude $m(i)$ and sign $s(i)$ sub-series are formed from the increments. If $\Delta x(i)=0, s(i)$ can be defined as $+1,0$ or -1 . In this paper we choose $s(0)=0$. Since there are not many zero-crossings in the geoelectrical time series analyzed, this choice does not alter the results obtained.

3. To avoid artificial trends we subtract from the magnitude and sign series their respective average values.

4. Since the DFA does not permit accurate estimation of scaling exponents of strong anticorrelated signals $(\alpha$ close to zero), we first integrate the sub-series.

5. The DFA is performed on the integrated sub-series. Then, the value obtained of the scaling exponent is $\alpha^{\prime}=\alpha+1$, where $\alpha$ is the scaling exponent of the original sub-series.

As an example, in Fig. 3 we show the original series $x(i)$ (a), the increment series $\Delta x(i)$ (b), the magnitude sub-series $m(i)$ (c) and the sign sub-series $s(i)$ (d) of a segment of Giul1 series.

Correlation in the magnitude series indicates that an increment with large (small) magnitude is more likely to be

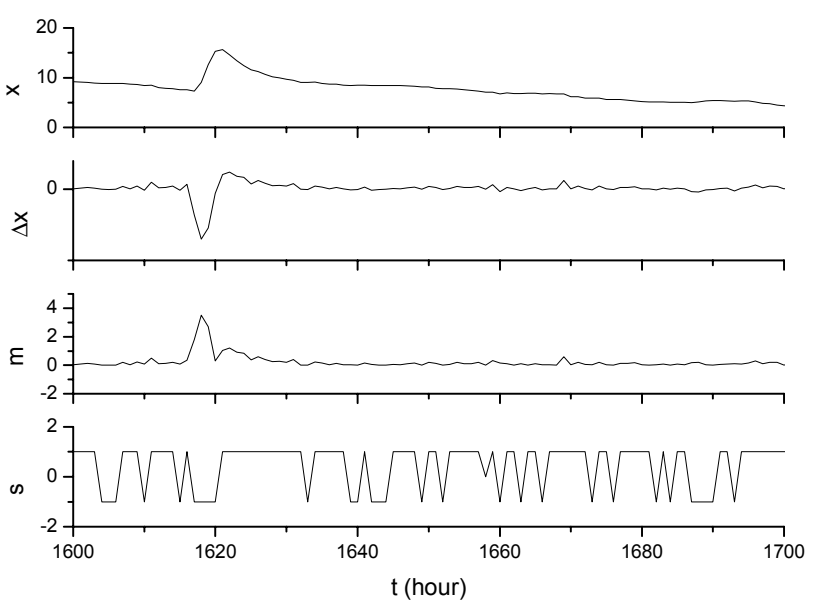

Fig. 3. Excerpt of Giull signal, with the increment, magnitude and sign series.

followed by an increment with large (small) magnitude. Anticorrelation in the sign series indicates that a positive increment is more likely to be followed by a negative increment and vice versa (Ashkenazy et al., 2003).

\section{Results}

The geoelectrical data present gaps; therefore, we considered for each signal the longest segments without data missings. The order of the magnitude of the length of each segment is about $10^{3}$, thus permitting to obtain reliable estimates of the scaling exponents. We could select 4 segments for all the signals except Tito, for which we were able to select only two segments. Figure 4 shows the DFA performed on all the segments of the original signals. The variation of the scaling exponent $\alpha$ is between 1.07 and 1.53. The average value of the scaling exponents is: 1.42 (Giul1), 1.41 (Giul2), 1.31 (Lat1), 1.26 (Lat2), 1.43 (Mar), 1.17 (Tit1), 1.22 (Tit2), 1.32 (Tit3) and 1.33 (Tit4). All the signals present close values of the scaling exponents that indicate the presence of persistent power-law correlated behaviour. From the values of the scaling exponents, estimated by the DFA applied to the original time series, we are not able to discriminate site dependencies; in fact, the signals measured at station Laterza, located in a low-seismicity area, show a mean scaling exponent comparable with those estimated for the remaining signals measured at stations installed in seismic areas.

We applied the decomposition method described in the previous section. For each signal segment, we created the increment series, then the magnitude and the sign sub-series. Thus we applied the DFA over all the sub-series following the steps 1)-5) outlined in the Sect. 4. Figure 5 shows the scaling exponents, $\alpha_{\text {mag }}$ and $\alpha_{\text {sig }}$, estimated for the magnitude and sign sub-series respectively, for each signal segment. We observe that the signals measured in seismic areas (Giul1, Giul2, Mar, Tit1, Tit2, Tit3 and Tit4) are on average characterized by a value of the scaling exponents $\alpha_{\mathrm{mag}}$ 

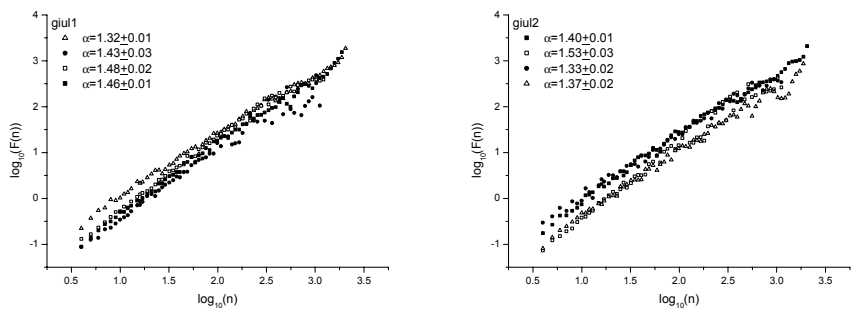

(a)

(b)
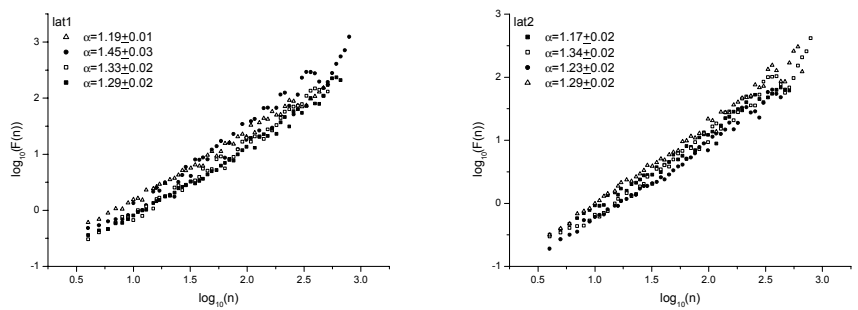

(c)

(d)
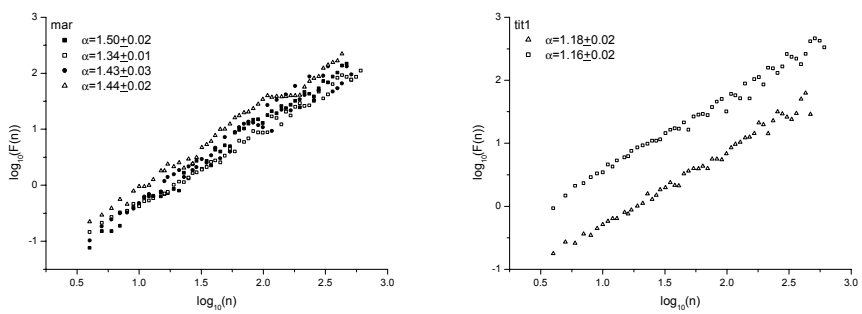

(e)
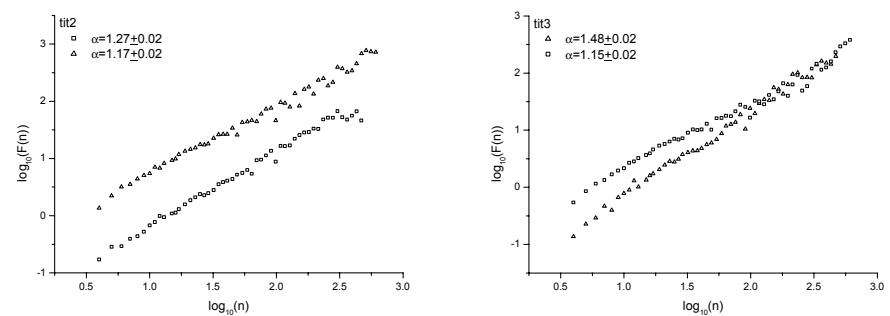

(g)

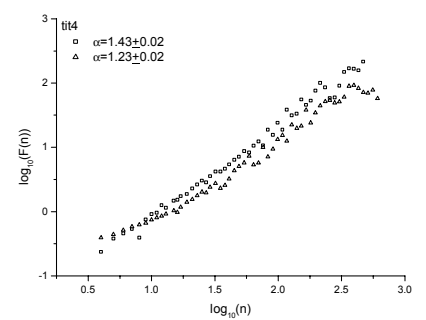

(i)

Fig. 4. DFA results for all the signal segments extracted from the geoelectrical time series.

and $\alpha_{\text {sig }}$ higher than those estimated for signals measured in low-seismicity areas (Lat1 and Lat2).

Scaling laws based on two-point correlation methods cannot inform about the nonlinearity of a series, but the twopoint correlations in the magnitude series reflect the nonlinearity of the original series (Ashkenazy et al., 2003). To verify this point, we generated surrogate series of the original increment time series, by firstly performing a Fourier transform on the time series, secondly preserving the amplitude but randomizing the phases (i.e. attributing to the phase a random number between 0 and $2 \pi$ ), and thirdly performing an inverse Fourier transform. The series generated in this manner has the same two-point correlations as the original one (the same power spectrum), but all the nonlinearities, stored in the phases, are eliminated by the randomisation. The difference between the exponents before and after the surrogate data test for nonlinearity may be quantified as follows. If $\alpha_{\mathrm{mag}, \text { sig }}$ is the exponent derived from the original magnitude 


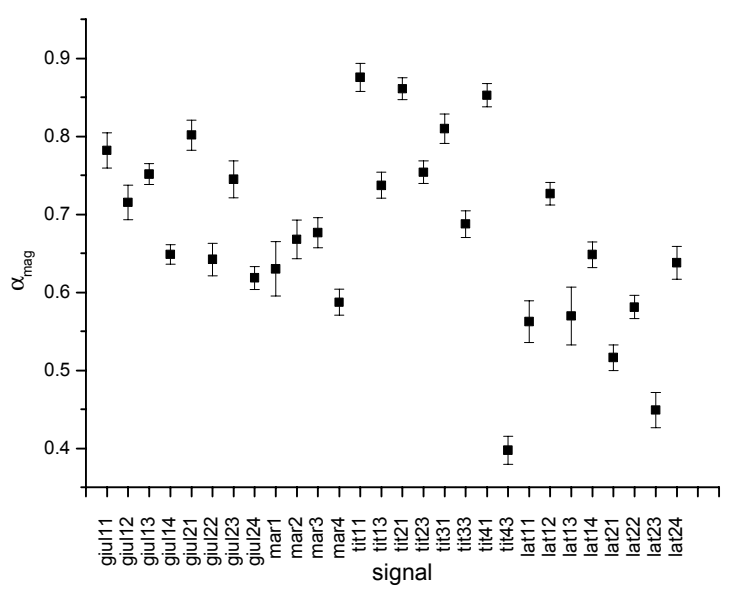

(a)

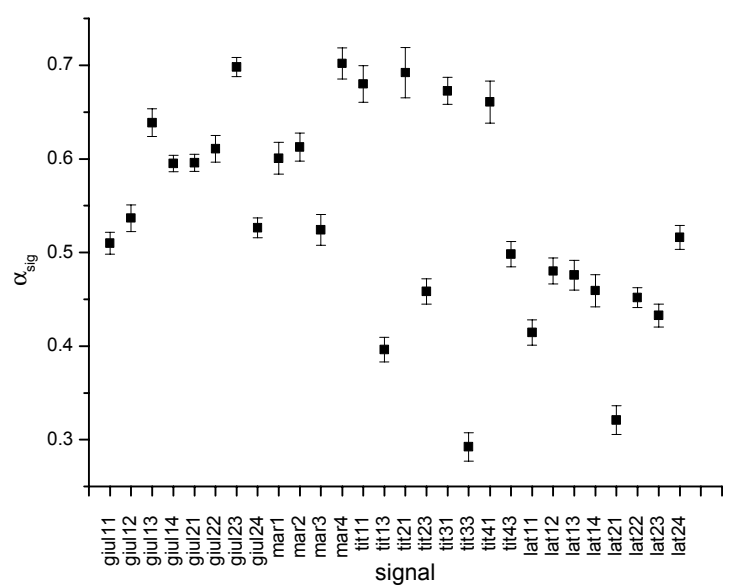

(b)

Fig. 5. Scaling exponents of the magnitude (a) and sign (b) subseries for each signal segment.

or sign sub-series and $\mu_{s}, \sigma_{S}$ are the average and standard deviation of the exponents derived from the surrogate data, then the separation is given by

$\sigma=\frac{\left|\alpha_{\text {mag, sig }}-\mu_{S}\right|}{\sigma_{S}}$,

where $\sigma$ measures how many standard deviations the original exponent is separated from the surrogate data exponent. The larger the $\sigma$ the larger the separation between the exponents derived from the surrogate data and the exponent derived from the original data. Thus, larger $\sigma$ values indicate stronger nonlinearity. The p-value is calculated by means of the formula $\mathrm{p}=\operatorname{erfc}(\sigma / \sqrt{ } 2)$ (Theiler et al., 1992); this is the probability of observing a $\sigma$ or larger if the null hypothesis is true, where the null hypothesis is given by the absence of nonlinearity. Figure 6 shows the $\sigma$ value for the magnitude and sign sub-series for each signal segment. It is also shown the horizontal dotted line, representing the threshold of $99 \%$ significance. Thus, values below this threshold indicate no statistically significant difference between original

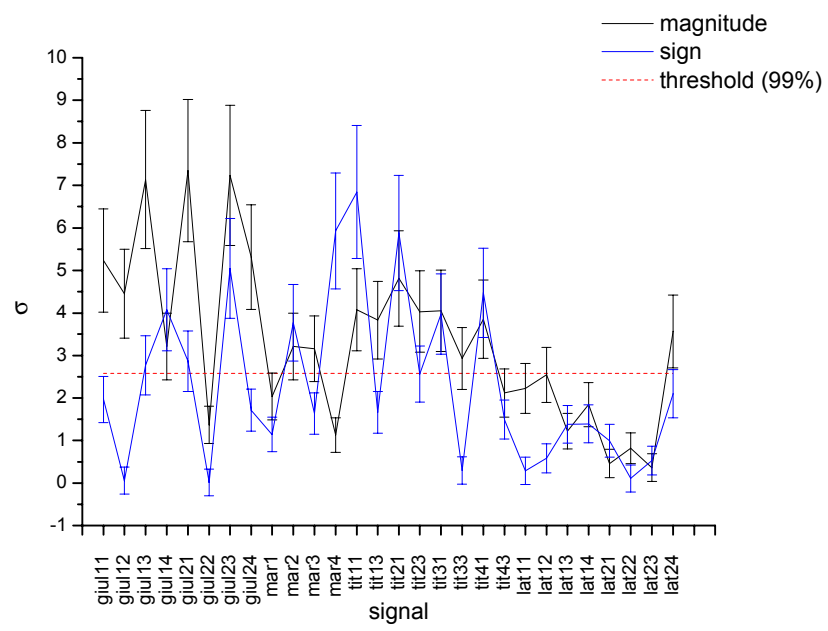

Fig. 6. 99\% significance analysis of the magnitude and sign scaling exponents.

data and surrogate data; in other word, if the $\sigma$ value is over the threshold, the corresponding signal has the probability of $99 \%$ to be characterized by nonlinear dynamics. We observe that Laterza $\sigma$ values are below the threshold regarding both the sign and the magnitude series (only lat24 has the magnitude exponent significantly different from the mean surrogate exponent). The other signals, measured in seismic areas, present magnitude $\sigma$ value above the threshold in most cases; while the sign $\sigma$ values above the threshold and those below are almost identically distributed. From this result, we deduce that the magnitude series convey information about the nonlinearity of the process underlying the signal variation. From these results we can observe that the signals measured in low-seismicity areas, like Laterza, are characterized by linear dynamics, while those measured in seismic areas by nonlinear dynamics.

But, a better discrimination between both classes of signals can be obtained plotting all the points representing the original data and the surrogate data in the $\alpha_{\mathrm{mag}}-\alpha_{\mathrm{sig}}$ plane. Figure 7 shows this relation for the original signals (circle) and surrogates (crosses) of Tito (a), Giuliano (b), Marsico (c) and Laterza (d). We can observe that the points representing the signals measured in seismic areas (Tito, Giuliano and Marsico) are well discriminated from those corresponding to the surrogates, while the points associated to the signals measured in low-seismicity areas (Laterza) are well mixed with the surrogates.

\section{Discussion and conclusions}

We analyzed the correlation properties of the magnitude and sign series of geoelectrical signals measured in seismic and low-seismicity areas. We conclude that signals with similar correlation properties (the DFA scaling exponents) can have different time ordering which can be characterized by different scaling exponents for the magnitude and sign se- 

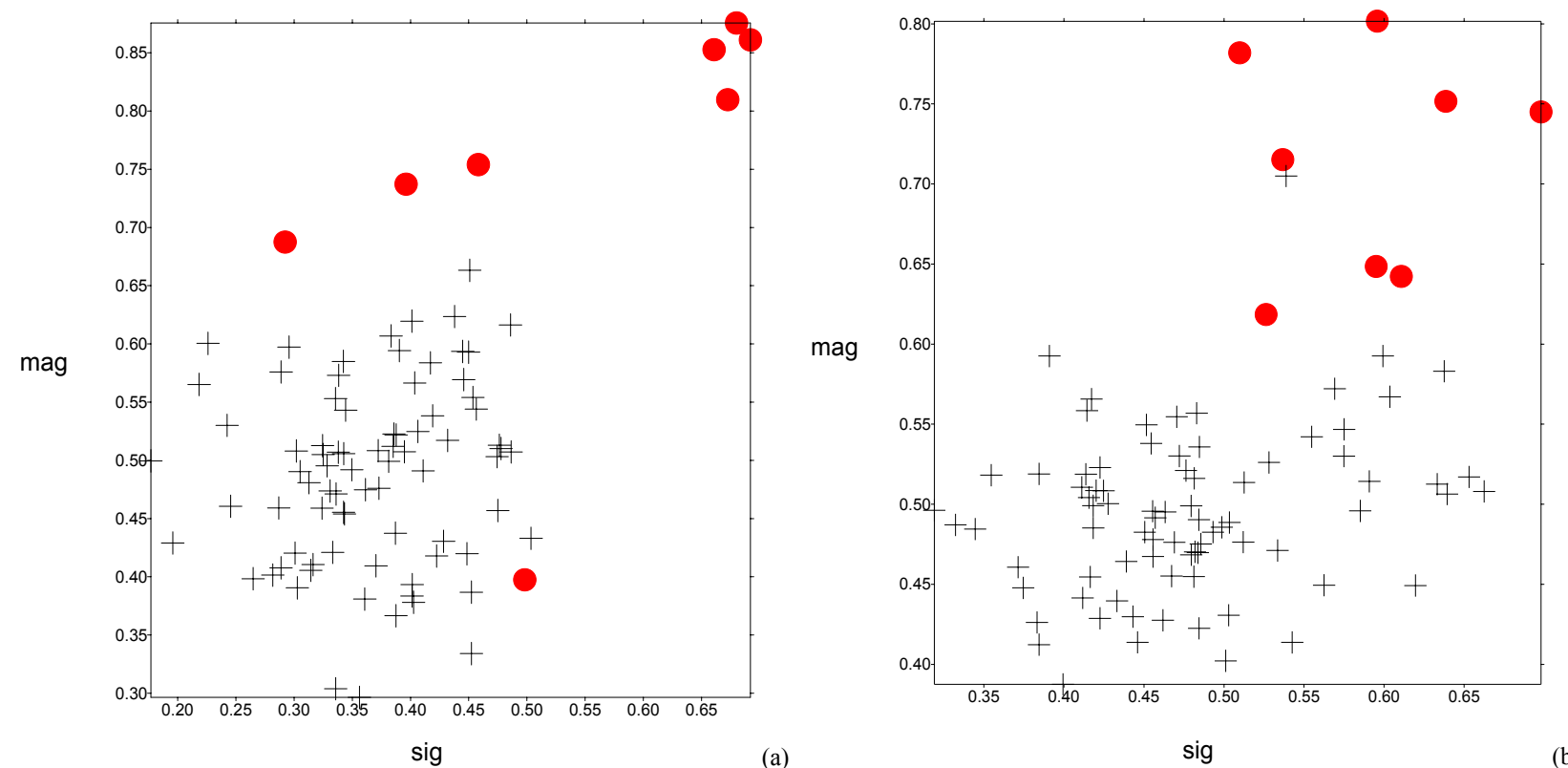

(a)

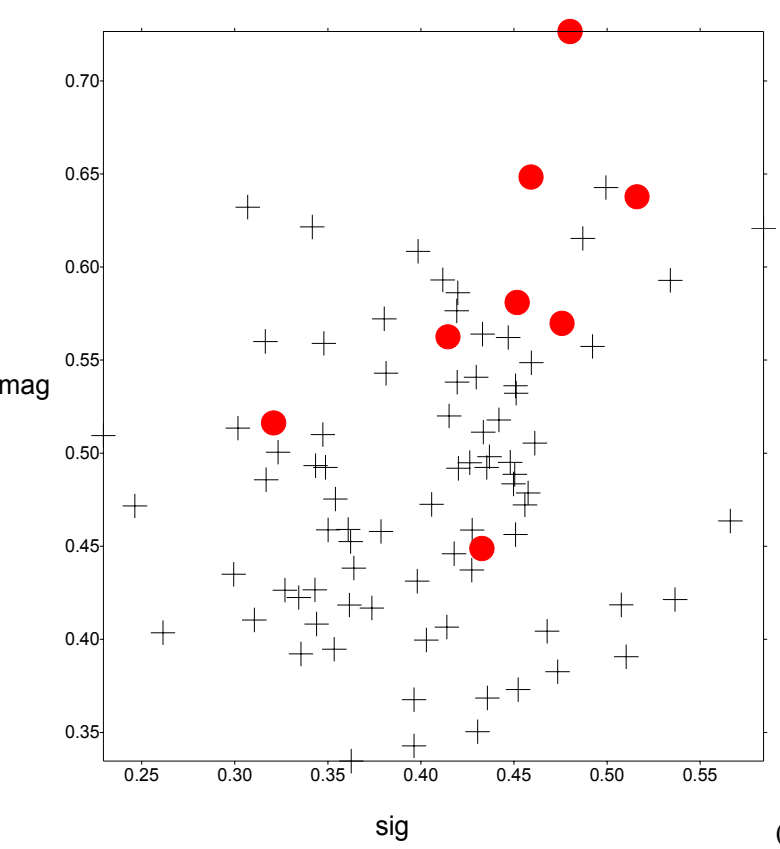

b)

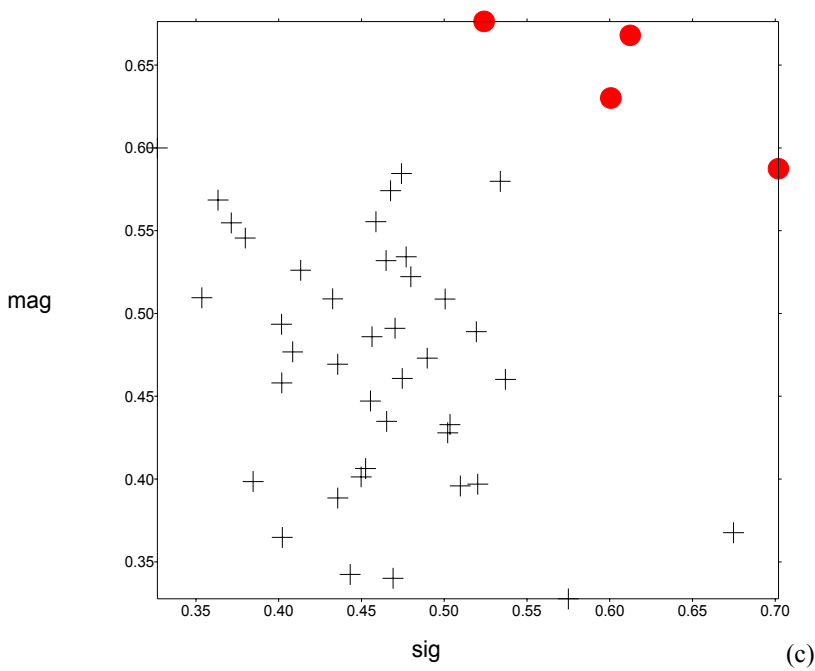

(c)

(d)

Fig. 7. $\alpha_{\text {mag }}-\alpha_{\text {sig }}$ plane representation of the signal segments along with their surrogates: (a) Tito, (b) Giuliano, (c) Marsico and (d) Laterza.

ries. The magnitude series reflects the way the series increments are clusterized, while the sign series reflects the way they alternate. Furthermore, the magnitude series conveys information about the nonlinearity of the original signals. In our analysis, we found that signals measured in seismic and low-seismicity areas show almost similar twopoint correlation properties, as indicated by the close values of the DFA scaling exponents, estimated on the original series. But, the decomposition between magnitude and sign sub-series and the analysis of their surrogate series have shown that the two classes of signals can reveal different properties in terms of nonlinearity. In particular, representing the signals and their surrogates in the $\alpha_{\mathrm{mag}}-\alpha_{\text {sig }}$ plane, we observe that signals measured in seismic areas are typically characterized by nonlinear dynamics (as shown by the clear separation between the points representing the original signals and those representing the surrogates), while signals measured in low-seismicity areas are characterized by linear dynamics (as shown by the mixing of the points representing original series as their surrogates). What is the geophysical meaning of nonlinear dynamics in signals recorded in seismic areas? The nonlinearity measured in the geoelectrical signals recorded in seismic areas can reflect the irregularity and heterogeneity of the crust, within which phenomena generating geoelectrical fields occur. In fact, it is well known that the most relevant phenomenon that could originate the 
geoelectrical field is the streaming potential: the electrical signal is produced, when a fluid flows in a porous rock due to a pore pressure gradient (Mizutani et al., 1976; Patella, 1997). In a seismic focal region the increasing accumulation of strain can cause dilatancy of rocks (Nur, 1972). Therefore the structure of the geoelectrical signal is linked to the structure of the seismic focal zone. Furthermore, the nonlinear character of geoelectrical signals generated in seismic areas could be related with the geometry and the structure of individual fault zones, that can be represented by a network with an anisotropic distribution of fracture orientations, and consisting of fault-related structures including small faults, fractures, veins and folds. This is a consequence of the roughness of the boundaries between each component and the interaction between the distinct components within the fault zone (O'Brien et al., 2003). In fact earthquake faulting is characterized by irregular rupture propagation and non-uniform distributions of rupture velocity, stress drop and co-seismic slip (Aki, 1979; Spudich and Frazer, 1984). These observations indicate a non-uniform distribution of strength in the fault zone, whose geometry and mechanical heterogeneities are important factors to be considered in the prediction of strong motion.

Acknowledgements. The authors are grateful to F. Vallianatos and an anonymous referee, whose suggestions significantly improved the present paper.

Edited by: P. F. Biagi

Reviewed by: F. Vallianatos and another referee

\section{References}

Alessio, G., Esposito, F., Gorini, A., and Porfido S.: Detailed study of the Potentino seismic zone in the Southern Apennines, Tectonophysics, 250, 113-134, 1995.

Ashkenazy, Y., Ivanov, P. Ch., Havlin, S., Peng, C.-H., Goldberger, A. L., and Stanley, H. E.: Magnitude and sign correlations in heartbeat fluctuations, Phys. Rev. Lett., 86, 1900-1903, 2001.

Ashkenazy, Y., Havlin, S., Ivanov, P. Ch., Peng, C.-K., SchulteFrohlinde, V., and Stanley, H. E.: Magnitude and sign scaling in power-law correlated time series, Physica A, 323, 19-41, 2003.

Cuomo, V., Lapenna, V., Macchiato, M., and Serio, C.: Autoregressive models as a tool to discriminate chaos from randomness in geoelectrical time series: an application to earthquake prediction, Annali di Geofisica, XL, 385-400, 1997.

Di Bello, G., Lapenna, V., Satriano C., and Tramutoli, V.: Selfpotential time series analysis in a seismic area of the Southern Apennines: preliminary results, Annali di Geofisica, XXXVII, Suppl. N. 5, 1137-1148, 1994.
Dobrovol'skiy, I. P.: Analysis of preparation of a strong tectonic earthquake, Phys. Solid Earth, 28, 481-492, 1993.

Dobrovol'skiy, I. P., Zubkov, S. I., and Miachkin, V. I.: Estimation of the size of earthquake preparation zones, Pageoph, 117, 10251044, 1979.

Ekström, G.: Teleseismic analysis of the 1990 and 1991 earthquakes near Potenza, Annali di Geofisica, 37, 1591-1599, 1994.

Johnston, M. J. S.: Review of electric and magnetic fields accompanying seismic and volcanic activity, S. Geophys., 18, 441-475, 1997.

Kantelhardt, J. W., Konscienly-Bunde, E., Rego, H. H. A., Havlin, S., and Bunde, A.: Detecting long-range correlations with Detrended Fluctuation Analysis, Physica A, 295, 441-454, 2001.

Giano, I., Maschio, L., Alessio, M., Ferranti, L., Improta, S., and Schiattarella, M.: Radiocarbon Dating of the active faulting in the Agri High valley, Southern Italy, J. Geodynamics, 29, 371386, 1999.

Martinelli, G. and Albarello, D.: Main constraints for siting monitoring networks devoted to the study of earthquake related hydrogeochemical phenomena in Italy, Annali di Geofisica, 40, 15051522, 1997.

Mizutani , H., Ishido, T., Yokokura, T., and Ohnishi, S.: Electrokinetic phenomena associated with earthquakes, Geophys. Res. Lett., 3, 365-368, 1976.

Monaco, C. and Tortorici, L.: Tectonic role of ophiolote-bearing terranes in the development of the Shouthern Apennines orogenic belt, Terra Nova, 7, 153-160, 1995.

Nur, A.: Dilatancy pore fluids and premonitory variations of ts/tp travel times, Bull. Seism. Soc. Am., 62, 1217-1222, 1972.

O'Brien,G. S., Bean, C. J., and McDermott, F.: Numerical investigations of passive and reactive flow through generic single fractures with heterogeneous permeability, Earth Planet. Sci. Lett. 213, 271-284, 2003.

Park, S. K.: Monitoring resistivity change in Parkfield, California: 1988-1995, J. Geophys. Res., 102, 24545-24559, 1995.

Patella D., Tramacere, A., and Di Maio, R.: Modelling earth current precursors in earthquake prediction, Annali di Geofisica, 40, 495-517, 1997.

Patella, D.: Introduction to ground surface self-potential tomography, Geophys. Prospect., 45, 653-681, 1997.

Peng, C.-K., Havlin, S., Stanley, H. E., and Goldberger, A. L.: Quantification of scaling exponents and crossover phenomena in nonstationary heartbeat time series, CHAOS, 5, 82-87, 1995.

Spudich, P. and Frazer, L. N.: Use of ray theory to calculate high frequency radiation from earthquake sources having spatially variable rupture velocity and stress drop, Bull. Seismol. Soc. Am., 74, 2061-2082, 1984.

Tertulliani, A., Anzidei, M., Maramai, A., Murru, M. and Riguzzi, F.: Macroseismic study of the Potenza (Southern Italy) earthquake of 5 May 1990, Natural Hazards, 6, 25-38, 1992.

Theiler, J., Eubank, S., Longtin, A., Galdrikian, B., and Farmer, J. D.: Testing for nonlinearity in time series: the method of surrogate data, Physica D, 58, 77-94, 1992. 